\title{
Diabetes in the elderly: is age a limiting control target?
}

\section{Summary}

Goals: To compare demographic, anthropometric and clinical characteristics in diabetic $>$ and $<65$ years and to determine the impact of age on the control target.

Methods: Observational, prospective and comparative study. Diabetic patients attended as outpatients were analyzed in Lagomaggiore Hospital, recorded in our database until August 2017. Patients with diabetes $>65$ years were compared with the rest of the diabetic population. Variables studied were: demographic, anthropometric, type of diabetes mellitus (DM), micro and macrovascular complications (MAP), compliance glycemic control targets (GCT), lipid (LipCT), blood pressure (BPCT), microalbuminuria and/or proteinuria (MPCT), current smoking (TABA). Analysis: MedCalc.

Results: 364 diabetic patients were included, $60(16.5 \%)$ had $>65$ years. Comparative analysis: Male $25(41.7 \%)$ vs $85(28.0 \%)$ p 0.04. >10 years of evolution: $41(68.3 \%)$ vs $108(35.6 \%) \mathrm{p}<0.001$. DM type2: $58(96.7 \%)$ vs $255(83.9 \%) \mathrm{p} 0.01$. Arterial hypertension (AH): $51(85 \%)$ vs $193(63.5 \%)$ p 0001. Acute myocardial infarction $15(25 \%)$ vs $17(5.6 \%)$ $\mathrm{p}<0.001$. Heart failure: $16(26.7 \%)$ vs $34(11.2 \%)$ p 0.001 . MAP: $20(33.2 \%)$ vs $39(12.8 \%)$ p $<0.001$. Retinopathy: $35(64.8 \%)$ vs. $144(52 \%)$ p 0.08 . Neuropathy: $15(25 \%)$ vs 45 (14.8\%) p 0.05. Chronic kidney disease state 3 and 4: $16(27.6 \%)$ vs $35(11.8 \%)$ p 002. BPCT: $49(81.7 \%)$ vs $252(82.9 \%)$ p 0.8. GCT: $33(55.9 \%)$ vs $137(46.6 \%)$ p 0.2. LipCT: $29(50.9 \%)$ vs. $144(49 \%)$ p 0.8. MPCT: $40(71.4 \%)$ vs $176(63.3 \%) 0.2$ p. TABA: $5(8.3 \%)$ vs $66(21.7 \%) 0.01 \mathrm{p}$.

Conclusions: Our diabetics patients $>65$ years had more comorbidities, cardiovascular risk factors and macrovascular complications. Despite this, there was no difference in achieving control target or medication use.

Keywords: diabetes mellitus, elderly, comorbid, medication, glycemic control targets, blood pressure, diabetic patients, heart failure
Volume 3 Issue 5 - 2019

\author{
Cynthia Antonella Anci, Guillermo Marcucci, \\ Vanina Solavallone, Hugo Lavandaio, Luz \\ Carolina Dromi \\ Internal Medicine Service, Lagomaggiore Hospital- Mendoza- \\ Argentina
}

Correspondence: $\mathrm{ANCl}$ Alvarez Cynthia Antonella, Internal Medicine Service, Lagomaggiore Hospital- Mendoza, Argentina, Leonidas Aguirre 1502-San Jose Guaymallen-Mendoza-Argentina. CP: 5519, Tel +54926I5I587I8,

Email ancicynthiaantonella@gmail.com

Received: September 07, 2019 | Published: October 03, 2019
Abbreviations: DM, diabetes mellitus; MAP, micro and macrovascular complications; GCT, glycemic control targets; LipCT, lipid; BPCT, blood pressure; MPCT, microalbuminuria and/or proteinuria; TABA, current smoking; GFR, glomerular filtration rate; $\mathrm{AH}$, Arterial hypertension

\section{Introduction}

The increase in life expectancy worldwide has allowed a rise in the incidence and prevalence of type 2 diabetes mellitus in the elderly; these being those over 65 years. ${ }^{1}$ This increment would respond to the aging of industrialized countries. ${ }^{2}$ Patients diagnosed at an early age have longer survival but also the diagnosis occurs at a higher age. ${ }^{3}$ Greater insulin resistance in the group is associated with greater sarcopenia, lower pancreatic endocrine functionality, lower physical activity and therefore more obesity, which could explain this situation. ${ }^{4}$ In USA, the prevalence of type 2 diabetes mellitus in older adults reaches $25 \%$, according to statistics from 2012; situation that considerably increases health costs. ${ }^{4,5,6}$ It is important to know if this group establishes different control challenges, develops more complications or requires different treatments than those established for younger diabetics. Therefore, we decided to study our population of elderly patients with diabetes mellitus, comparing them in our database with the rest of our diabetic population.

\section{Material and methods}

Observational, prospective and comparative study was made 364 outpatient diabetic patients were registered in our database from August 2015 to August 2017, in an acute hospital. Diabetic patients over 65 years of age were compared with the rest of the diabetic population in the sample. Demographic and anthropometric variables were evaluated, as well as type of diabetes mellitus and its evolution time. Micro and macroangiopathic complications, compliance with glycemic, lipid control, blood pressure and microalbuminuria and/ or proteinuria control targets were also included in the analysis. In addition, the history of current smoking was evaluated. The glomerular filtration rate (GFR) was calculated to determine chronic kidney disease in its different stages, according to the MDRD4 formula. Statistical analysis was performed using SPSS v20: measures of central tendency, dispersion $(95 \% \mathrm{CI})$, chi2 and Student test, significance criterion error $\alpha<5 \%$.

\section{Results}

364 diabetic patients were analyzed, of which 60 patients were over 65 years $(16.5 \%)$. In the comparative analysis of the group older than 65 years versus (vs) the group of 65 years or less, the results were: The male gender was found in 25 patients $(41.7 \%)$ of the first group vs 85 (28\%) of the second group, p 0.04. Patients with Type 2 Diabetes Mellitus were: 58 (96.7\%) and 255 (83.9\%), respectively, p 
0.009; having an evolution greater than 10 years 41 (68.3\%) vs 108 $(35.6 \%), \mathrm{p}<0.001$. Comorbidities analyzing, arterial hypertension: 51 (85\%) vs 193 (63.5\%), p 0.001, acute previous myocardial infarction: $15(25 \%)$ vs $17(5.6 \%), \mathrm{p}<0.001$, heart failure: $16(26.7 \%)$ vs $(6.6 \%)$, p 0.001 . Development of transient ischemic accident $3(5 \%)$ vs 1 $(0.3 \%)$, in each group, p 0.002. Current smoking: 5 (8.3\%) and 66 (21.7\%), respectively, p 0.01. Macroangiopathic complications were: $20(33.2 \%)$ vs. 39 (12.8\%), p<0.001 (Table 1) \& (Table 2). Diabetic retinopathy 35 patients $(64.8 \%)$ over 65 years compared to $144(52 \%)$ under 65 years, $\mathrm{p}$ 0.08. Diabetic neuropathy $15(25 \%)$ and $45(14.8 \%)$, respectively, p 0.05 and erectile dysfunction $19(31.7 \%)$ vs 41 (13.5\%), p 0.001 . When analyzing the development of chronic kidney disease, we observed that they had stages 3 and $4,16(27.6 \%)$ vs $35(11.8 \%)$, p 0.02 and non-proteinuric nephropathy $11(19.6 \%)$ and $13(11 \%)$, $\mathrm{p}<0.001$ with the same stadiums. The value of the glomerular filtration rate was: $74.35(+25.34) \mathrm{ml} / \mathrm{min}$ vs. $87.56(+26.1) \mathrm{ml} / \mathrm{min}, \mathrm{p}<0.001$. Fatty liver was diagnosed in $4(9.5 \%)$ vs $66(59.2 \%)$, p 0.009 .

Table I Population characteristics

\begin{tabular}{llll}
\hline Variable & $>65$ years & $\leq 65$ years & $\mathbf{p}$ \\
\hline Age & $71.6(+5.4)$ & $71.6(+5.4)$ & $<0.00 \mathrm{I}$ \\
Male & $25(41.7 \%)$ & $85(28 \%)$ & 0.035 \\
More 10 years of evolution & $41(68.3 \%)$ & $108(35.6)$ & $<0.00 \mathrm{I}$ \\
DM type 2 & $58(96.7 \%)$ & $255(83.9 \%)$ & 0.009 \\
BMI & $30.7+5.2$ & $33.8+7.4$ & 0.02 \\
Waist perimeter & $102.5+11.6$ & $105.9+16.1$ & 0.02 \\
UKPDS Risk & $30.2+17.3$ & $12.1+9.3$ & $<0.00 \mathrm{I}$ \\
Fatty liver & $4(9.5 \%)$ & $66(59.2 \%)$ & 0.009
\end{tabular}

Table 2 Comorbidities

\begin{tabular}{llll}
\hline Variable & $>65$ years & $\leq 65$ years & $\mathbf{p}$ \\
Arterial hypertension & $51(85 \%)$ & $193(63.5 \%)$ & $0.00 \mathrm{I}$ \\
Acute myocardial infarction & $15(25 \%)$ & $17(5.6 \%)$ & $<0.00 \mathrm{I}$ \\
Coronary bypass & $4(6.7 \%)$ & $3(1 \%)$ & 0.003 \\
Heart failure & $16(26.7 \%)$ & $34(11.2 \%)$ & 0.001 \\
Intermittent claudication & $12(20 \%)$ & $20(6.6 \%)$ & 0.001 \\
Transient ischemic stroke & $3(5 \%)$ & $1(0.3 \%)$ & 0.002 \\
\hline
\end{tabular}

The analysis of the control target was: blood pressure control was $49(81.7 \%)$ vs $252(82.9 \%)$, p 0.81 . Glycemic: $33(55.9 \%)$ vs 137 (46.6\%), p 0.19. Lipid control: 29 (50.9\%) vs 144 (49\%), p 0.8 and microalbuminuria or proteinuria: $40(71.4 \%)$ vs $176(63.3 \%)$, p 0.24 . Four targets were met without current smoking: $15(25 \%)$ vs 49 (16.1\%), p 0.09. Body mass index was $30.7(+5.3)$ vs $33.8(+7.4)$, p 0.02 , while waist circumference was $102.5(+11.6)$ vs $105.9(+16.1)$, p 0.02 (Table 3). The UKPDS Risk calculation showed $30.2(+17.3)$ vs. $12.1(+9.3), p<0.001$. No differences were found in the use of metformin, sulfonylureas or insulin. In the group older than 65 years, patients with less than 5 years of evolution of diabetes were analyzed, compared with those with more than 5 years. The results were: male gender $9(56.3 \%)$ vs $16(36.4 \%)$, p 0.17 , age at diagnosis $67.1(+7)$ vs. $53.3(+10.8)$, p 0.000 . The evolution time of diabetes was 3.2 $(+2.1)$ and $18.5(+8.2)$, respectively, p0, 000. Comorbid: arterial hypertension $12(75 \%)$ vs 39 (86.6\%), p 0.19, dyslipidemia 8 (50\%) vs $36(81.8 \%)$, p 0.014, acute myocardial infarction 3 (18.8\%) vs 12
(27.3\%), p 0.5, chronic heart failure 4 (25\%) vs 12 (27.3\%), p 0.86 and intermittent claudication $0(0 \%)$ vs $12(27.3 \%)$, p 0.02. Regarding to macroangiopathic complications, $5(31.3 \%)$ and $15(34.1 \%)$ were detected, p 0.84, diabetic retinopathy in $7(50 \%)$ and $28(70 \%), \mathrm{p} 0.18$. Stage 3 and 4 chronic kidney disease, 1 (6.7\%) vs 15 (34.9\%), p 0.03 . Four control targets in non-smokers in $4(25 \%)$ vs 13 (29.5\%), p 0.73 (Table 4).

Table 3 Microangiopathic Complications

\begin{tabular}{llll}
\hline Variable & $>65$ years & $\leq 65$ years & $\mathbf{P}$ \\
Microangiopathic complications & $20(33.2 \%)$ & $39(12.8 \%)$ & $<0.00 \mathrm{I}$ \\
Diabetic retinopathy & $35(64.8 \%)$ & $144(52 \%)$ & 0.084 \\
Diabetic neuropathy & $15(25 \%)$ & $45(14.8 \%)$ & 0.052 \\
Erectile dysfunction & $19(31.7 \%)$ & $41(13.5 \%)$ & $0.00 \mathrm{I}$ \\
Stage 3 and 4 nephropathy & $16(27.6 \%)$ & $35(11.8 \%)$ & 0.002 \\
Non-proteinuric nephropathy & $\mathrm{II}(19.6 \%)$ & $13(11 \%)$ & $<0.00 \mathrm{I}$ \\
\hline
\end{tabular}

Table 4 Control target

\begin{tabular}{llll}
\hline Variable & $>\mathbf{6 5}$ years & $\leq \mathbf{6 5}$ years & $\mathbf{P}$ \\
\hline GCT & $33(55.9 \%)$ & $137(46.6 \%)$ & 0.2 \\
HbAIlc $\dot{x}$ & $7.62 \%(+2.1)$ & $7.8 \%(+2.04)$ & 0.6 \\
LipCT & $29(50.9 \%)$ & $144(49 \%)$ & 0.8 \\
BPCT & $49(81.7 \%)$ & $252(82.9 \%)$ & 0.8 \\
MPCT & $40(71.4 \%)$ & $176(63.3 \%)$ & 0.2 \\
GFR (ml/min) & $74.4(+25.3)$ & $87.6(+26.1)$ & $<0.001$ \\
4 target + No TABA & $15(25 \%)$ & $49(16.1 \%)$ & 0.09 \\
\hline
\end{tabular}

\section{Discussion}

The concept of type 2 diabetes mellitus in the elderly is becoming more frequent. According to published studies, the prevalence of this situation reaches $25 \% ; ;^{4,5,6,7}$ slightly higher than estimated in our work, in which it was less than $20 \%$. There are few jobs that include this age group in the study population. Moreover, important studies such as the UK Prospective Diabetes Study exclude them from their analysis. As a result, there are some randomized controlled studies focused on glycemic control as well as the prevention of cardiovascular impact in both young and elderly adults. ${ }^{4}$ The management of these patients proposes constant questions and challenges, not only because of the greater frequency of comorbidities but also because of the associated cognitive impairment, polypharmacy associated with greater complications due to the added adverse effects and the presence of depression. ${ }^{1,4,5}$ Cardiovascular risk is higher in this group of patients, even double it according to some authors. ${ }^{2,4,6,7}$ In our study we evidenced this through a higher frequency of acute myocardial infarction, heart failure, need for coronary bypass, arterial hypertension, intermittent claudication and transient ischemic accident, with statistically significant results. Atherosclerotic disease is greater in elderly diabetics in relation to younger patients as well as in relation to elderly non-diabetic. . $^{3,8}$

Reduction of cardiovascular risk with the management of blood pressure, use of antiplatelet drugs, strict glycemic control or pharmacological management of lipids are unquestionable measures in young people but controversial in elderly patients, due to the increase in adverse effects and mortality. ${ }^{2,3,8}$ For this, it is very 
important to know the performance status of our elderly patients, as well as the fragility of the sample with which we work to avoid iatrogenic complications. ${ }^{8}$ Increase in micro and macroangiopathic complications observed in diabetic elderly patients, as published in the literature, ${ }^{2,3,9}$ we have evidenced in our study, being statistically significant erectile dysfunction, nephropathy in all stages both proteinuric and not proteinuric and diabetic neuropathy. Population older than 65, and especially the group comprised of those over 80, constitute a high-risk group, with a high fragility, in which the side effects or drugs are much more frequent. This is demonstrated by the high frequency of hypoglycemia associated with strict hypoglycemic treatments in these patients. ${ }^{9}$ In our study, the records of severe hypoglycemia between the two populations had no statistically significant differences.

Sarcopenia, considered as one of the changes in body composition that predisposes to insulin resistance in this age group ${ }^{4}$ is a parameter to consider. In our work, this could be represented by the lower body mass index in our study group, which was statistically significant. A finding in our elderly patient population is the lowest proportion of fatty liver. That could also be correlated with the low body mass index. However, it is known than there's a low correlation of the ultrasound scan and presence of fatty liver and fibrosis by biopsy. Currently, two different groups of diabetic elderly are considered, in terms of chronic complications and prognosis, those who are diagnosed at the age of 65 years and those who reach 65 years with diabetes mellitus with more than 10 years of evolution. In our population over 65 years of age, we compare those with less than 5 years of evolution versus those with more than 5 years. We found a higher frequency of lower limb claudication and chronic kidney disease in advanced stages in the group with the longest evolution time. There were no differences in overall macrovascular involvement or the presence of retinopathy. The achievement of control target did not show differences. It is important to establish that the time of evolution of the disease often does not match with the time of diagnosis, especially in a marginal urban population as is the reference of our hospital. It is increasingly common to meet diabetic patients over 65 years of age, resulting in a challenge for the physician both because of the pathology itself and the associated comorbidities and complications that result from their management. The need for scientific research that focuses on these situations, such as the development of guides adapted, is of vital importance to achieve successful results. A multidisciplinary work that includes both the pharmacological and social and psychological approach of these patients is essential for the success sought.

\section{Conclusion}

More comorbidities associated with more cardiovascular risk factors and macrovascular complications were found in the group of diabetics older than 65 years, compared to the group of younger diabetic patients. They presented more frequently stage 3 and 4 nephropathy and non-proteinuric nephropathy, especially in the subgroup with longer evolution of the disease. Despite this greater degree of fragility defined by its comorbidities and complications, there were no statistically significant differences in the achievement of control target or in the use of medication. Age did not limit the therapeutic efforts in our sample, nor conditioned the achievement of objectives.

\section{Acknowledgments}

None.

\section{Funding details}

None.

\section{Conflicts of interest}

The author declares there is no conflict of interest.

\section{References}

1. Meneilly GS, Knip A, Tessier D. Diabetes in the Elderly. Can J Diabetes. 2013;37(Suppl 1):184-190.

2. Kalyani R, Golden S, Cefalu W. Diabetes and Aging: Unique Considerations and Goals of Care. Diabetes Care. 2017;40(4):440-443.

3. Lee P, Halter J. The Pathophysiology of Hyperglycemia in Older Adults: Clinical Considerations. Diabetes Care. 2017;40(4):444-452.

4. Kirkman M, Jones Briscoe V, Clark N, et al. Diabetes in Older Adults Diabetes Care. 2012;35(12):2650-2664.

5. Munshi M, Florez H, Huang E, et al. Management of Diabetes in Longterm Care and Skilled Nursing Facilities: A Position Statement of the American Diabetes Association. Diabetes Care. 2016;39(2):308-318.

6. American Diabetes Association. Older Adults. Diabetes Care 2016;39(1):81-85

7. Sinclair AJ, Paolisso G, Castro M, et al. European Diabetes Working Party for Older People 2011 Clinical Guidelines for Type 2 Diabetes Mellitus. Executive summary. Diabetes Metab. 2011;37(Supple 3):27-38.

8. Korytkowski M, Forman D. Management of Atherosclerotic Cardiovascular Disease Risk Factors in the Older Adult Patient with Diabetes. Diabetes Care. 2017;40(4):476-484.

9. Abbatecola A, Paolisso G, Sinclair AJ. Treating Diabetes Mellitus in Older and Oldest Old Patients. Curr Pharm Des. 2015;21(13):1665-1671. 